\title{
A Goldstone Theorem in Thermal Relativistic Quantum Field Theory
}

\author{
Christian D. Jäke* \\ School of Mathematics, Cardiff University, Wales, \\ CF24 4AG, United Kingdom. \\ Walter F. Wreszinsk 0 i \\ Departamento de Física Matemática, Instituto de Física, \\ USP, Caixa Postal 66318 05314-970, São Paulo, Brazil.
}

\begin{abstract}
We prove a Goldstone Theorem in thermal relativistic quantum field theory, which relates spontaneous symmetry breaking to the rate of space-like decay of the two-point function. The critical rate of fall-off coincides with that of the massless free scalar field theory. Related results and open problems are briefly discussed.
\end{abstract}

PACS numbers: 81T08, 82B21, 82B31, 46L55

Keywords: Goldstone theorem, thermal field theory, KMS states.

\section{INTRODUCTION AND SUMMARY}

Thermal quantum field theory ( $t q f t$ ) has received considerable attention recently, both from the conceptual and the constructive point of view (see [1] for a review and references). Its range of applications extends from heavy ion collisions and cosmology at early stages (see [2] for a review) to the present day hot big-bang model in cosmology [3], with obvious potential relevance to the dark energy problem [4], which, however, remains to be explored.

In the present paper, we study the spontaneous symmetry breaking ( $s s b)$ of continuous (internal) symmetries in relativistic thermal quantum field theory, and prove a version of Goldstone's theorem (see, e.g., 5] for a review and references) - Theorem III.3 of Section III — which relates $s s b$ to the asymptotic decay of (truncated) correlation functions for large space-like distances. In this respect the theorem follows the lines of 6 and 7], the latter having been proved to be an optimal version, generalizing the well-known Mermin-Wagner theorem of quantum statistical mechanics [8]. If, however, one endeavors to understand the concept and structure of particles in tqft, large time-like distances necessarily come into play, and in this connection the Goldstone-type theorem of Bros and Buchholz [9] is more natural (see Remark [II.1 of Section III and Section IV - discussion and outlook).

The main advantage of our approach lies in the possibility of a sharp distinction between massive and zeromass theories in terms of their correlation functions' rate of space-like decay (Conjecture III.4 of Section III): if such is true, a theorem of the same form as the vacuum ( $\mathrm{T}=0$, zero density) version [10, 11] follows (Corollary III.5) and Theorem III.3 turns out to be optimal as in the quantum statistical mechanical case.

Our proof of Theorem III.3 generalises the method

\footnotetext{
* christian.jaekel@mac.com

$\dagger$ wreszins@gmail.com, supported in part by CNPq
}

used in [6, 7], which was based on the Bogoliubov inequality (see [12] and the references given there), in an essential way: firstly, the treatment of the middle term in that inequality (see (82) of Appendix A) relies on local current conservation, Einstein causality and the definition (12) of the global charge, in a manner reminiscent of [13]; secondly, unlike in [6, 7], we employ a form of the Bogoliubov inequality which was proved to follow from the KMS condition for infinite systems by Garrison and Wong [14] in a $C^{*}$-algebraic framework. This naturally takes into account the singular nature of the quantum fields, which reflects itself in the necessity of choosing adequate test functions. For other related derivations, see [15] and [16], and [17], Vol. II, pg. 334.

We now briefly describe the organisation of the paper. In Section II we introduce the framework, which is that of $C^{*}$-dynamical systems (see e.g. 25]) and formulate our assumptions, together with some auxiliary lemmas. In Section III we prove our main result (Theorem III.3), followed by Conjecture III.4 and Corollary III.5 referred to before. The connection to $W^{*}$-dynamical systems and the spectral properties of the Liouvillian is also discussed there. Section IV is reserved for a discussion and outlook, in particular the relation to other approaches and open problems. In Appendix A we state Bogoliubov's inequality, with some additions needed in the main text. In Appendix B we state, for the reader's convenience, the theorem on the partition of unity used in the main text.

\section{FRAMEWORK AND ASSUMPTION}

We work in the framework of $C^{*}$-dynamical systems, consisting of a pair $(\mathcal{A}, \tau)$, where $\mathcal{A}$ is a $C^{*}$-algebra with unit and $\left\{\tau_{t}\right\}_{t \in \mathbb{R}}$ is a one-parameter group of norm continuous (time translation) automorphisms of $\mathcal{A}$ (see, e.g., 25]). Since the time-translation automorphisms are not norm continuous on the Weyl algebra (see [17], Vol. 2, Theorem 5.2.8), we adopt Haag's construction (see [18], pp. 129 et seq.), which leads to the following structure (see [18], III.3.3., pg. 141) - $\mathcal{O}$ denotes a finite, con- 
tractible, open region in Minkowski space:

(i) a net of $C^{*}$-algebras with common unit

$$
\mathcal{O} \rightarrow \mathcal{A}_{S}(\mathcal{O})
$$

with the total $C^{*}$-algebra (the $C^{*}$-inductive limit [19]) $\mathcal{A}_{S}$ :

$$
\mathcal{A}_{S}=\overline{\mathcal{A}_{L}}, \quad \text { with } \quad \mathcal{A}_{L}=\bigcup_{\mathcal{O}} \mathcal{A}_{S}(\mathcal{O}),
$$

where the bar denotes the completion in the norm topology. We call $\mathcal{A}_{L}$ the (strictly) local algebra. The action of the time automorphism $\left\{\tau_{t}\right\}_{t \in \mathbb{R}}$ on $\mathcal{A}_{S}$ is $t$-continuous in the norm topology (in [18], this is required for the space-time automorphisms, but we do not need the smoothness with respect to spatial translations);

(ii) a set $\mathcal{S}$ of physical (i.e., locally normal [18, 19]) states over $\mathcal{A}_{S}$ and the complex linear span of $\mathcal{S}$, denoted by $\Sigma$;

(iii) the dual of $\Sigma$ is a net of $W^{*}$-algebras with common unit

$$
\mathcal{O} \mapsto \mathcal{R}(\mathcal{O})=\Sigma(\mathcal{O})^{*}
$$

$\mathcal{R}(\mathcal{O})$ is closed in the weak topology induced by $\Sigma$ and $\mathcal{A}_{S}(\mathcal{O})$ is weakly dense in $\mathcal{R}(\mathcal{O})$.

(iv) Local commutativity: if the regions $\mathcal{O}_{1}$ and $\mathcal{O}_{2}$ are totally space-like to one another, then

$$
[A, B]=0 \quad \forall A \in \mathcal{A}\left(\mathcal{O}_{1}\right), \quad \forall B \in \mathcal{A}\left(\mathcal{O}_{2}\right) .
$$

We must also assume certain global properties on the particular state $\omega \in \mathcal{S}$ we shall work with. The basic property of thermal states is the KMS condition (see [17], Vol. 2):

Definition II.1 A state $\omega\left(=\omega_{\beta}\right)$ over $\mathcal{A}\left(=\mathcal{A}_{S}\right)$ is called a KMS state for some $\beta>0$, if for all $A, B \in \mathcal{A}$ there exists a function $F_{A, B}$, which is continuous in the strip $0 \leq \Im z \leq \beta$ and analytic and bounded in the open strip $0<\Im z<\beta$, with boundary values given by

$$
F_{A, B}(t)=\omega\left(A \tau_{t}(B)\right)
$$

and

$$
F_{A, B}(t+i \beta)=\omega\left(\tau_{t}(B) A\right)
$$

for all $t \in \mathbb{R}$.

We further assume that

A1 $\omega$ is a factor (primary) state over $\mathcal{A}_{S}$;

A2 $\omega$ satisfies the KMS condition.
From A2 it follows that $\omega$ is invariant under time translations, but we also need that

A3 $\omega$ is invariant under space translations.

By A2, A3 and the GNS construction there exists a representation $\pi_{\omega}$ of $\mathcal{A}_{S}$ on a Hilbert space $\mathcal{H}_{\omega}$, with selfadjoint space-time translation generators $\left(L_{\omega}, \vec{P}_{\omega}\right)$ and cyclic vector $\Omega_{\omega}$ such that

$$
L_{\omega} \Omega_{\omega}=0
$$

and

$$
\vec{P}_{\omega} \Omega_{\omega}=\overrightarrow{0}
$$

As occurs with $W^{*}$-dynamical systems, $L_{\omega}$ is not bounded below, see (74) et seq.. Of primary concern to us will be continuous internal symmetries generated by a local current $J_{\mu}(x)$ on which we make the same assumptions as in [10] (see p. 110), headed there under local current conservation. Before stating them we remark that in the following, when $A \in \mathcal{A}_{L}$ occurs in connection with the representation $\pi_{\omega}$, it is understood as $\pi_{\omega}(A)$. The assumptions are: there exist for every test-function $f \in \mathcal{D}=C_{0}^{\infty}\left(\mathbb{R}^{s+1}\right)$ a set of $(s+1)$ unbounded self-adjoint operators $J_{\mu}(f)$, with the properties

A4 $\Omega_{\omega}$ is in the domain of $J_{\mu}(f)$ for all $f \in \mathcal{D}$;

A5 $T(a) J_{\mu}(f) T(a)^{-1}=J_{\mu}\left(f_{a}\right)$ where $f_{a}(x)=f(x-a)$;

A6 $\sum_{\mu=0}^{s} J_{\mu}\left(\frac{\partial f}{\partial x_{\mu}}\right)=0 ;$

A7 (a) $\left(\Omega_{\omega},\left[J_{\mu}(f), A\right] \Omega_{\omega}\right)=0$ for $A \in \mathcal{A}_{S}(\mathcal{O})$, if the support of $f$ is totally space-like to $\mathcal{O}$;

(b) $\left(\Omega_{\omega},\left[J_{0}(f), \vec{J}(g)\right] \Omega_{\omega}\right)=\overrightarrow{0}$, if the supports of $f$ and $g$ are space-like to one another;

A8 for all $f \in \mathcal{D}$, the charge operator $J_{0}(f)$ is affiliated to $\mathcal{R}(\mathcal{O})$.

In $\mathbf{A 7}$ (a) the natural definition

$$
\begin{aligned}
\left(\Omega_{\omega},\left[J_{\mu}(f), A\right] \Omega_{\omega}\right)=\left(J_{\mu}(f) \Omega_{\omega}, A \Omega_{\omega}\right) & \\
& -\left(A^{*} \Omega_{\omega}, J_{\mu}(f) \Omega_{\omega}\right)
\end{aligned}
$$

is adopted. By $\mathbf{A} 4$ and (9), the term

$$
\left(\Omega_{\omega},\left[J_{0}(f), \vec{J}(g)\right] \Omega_{\omega}\right)
$$

is well-defined for all $f, g \in \mathcal{D}$. $\quad \mathbf{A 7}(\mathrm{b})$ follows from the assumption e.) of [10] (see pg. 110) that $\left(\Omega_{\omega},\left[J_{0}(f), \vec{J}(g)\right] \Omega_{\omega}\right)$ is a tempered distribution, but we only need $\mathbf{A} \mathbf{7}(\mathrm{b})$.

Assumption $\mathbf{A} 8$ had to be imposed on $J_{\mu}(f)$, because the KMS condition (5) a priori holds only for $A, B \in \mathcal{A}$. Recall that $\mathcal{R}(\mathcal{O})$ is the von Neumann algebra defined in (3), and the concept of affiliation is defined in [17] (see Vol. 1, Definition 2.5.7, pg. 87). By self-adjointness 
of $J_{0}(f)$ and [17], Lemma 2.5.8 (see Vol. 1, pg. 87) the spectral projections $E(\lambda)$ of $J_{0}(f)$ lie in $\mathcal{R}(\mathcal{O})$. Note that it is too much to require that they lie in $\mathcal{A}_{S}(\mathcal{O})$ : bounded functions of the fields are expected to belong only to the weak closure of the Weyl algebra, and are thus not smooth elements.

We have now completed our list of assumptions, and turn to our criterion of existence of $s s b$, which is the same as the one adopted in [10]. One might expect that the limit $V \rightarrow \infty$ of the local integrated current density

$$
\int_{V} \mathrm{~d}^{s} \vec{x} J_{0}\left(x_{0}, \vec{x}\right)
$$

defines a global charge operator, which serves as the generator of the internal symmetry considered. However, the limit $V \rightarrow \infty$ of (11) does not exist due to vacuum fluctuation occurring all over space, by translation invariance: this is, in fact, as remarked in [13], the source of $s s b$. We therefore define, as in [10], the charge operator corresponding to $J_{\mu}$ as a suitable limit of the operators

$$
J_{0}\left(f_{d} \otimes g_{R}\right):=\int \mathrm{d}^{s+1} x f_{d}\left(x_{0}\right) g\left(\frac{\vec{x}}{R}\right) J_{0}(x)
$$

as $R \rightarrow \infty$, where

$$
\begin{gathered}
g \in \mathcal{D}_{s}:=C_{0}^{\infty}\left(\mathbb{R}^{s}\right), \\
f_{d} \in \mathcal{D}:=C_{0}^{\infty}(\mathbb{R}), \\
\int \mathrm{d} x_{0} f_{d}\left(x_{0}\right)=1, \\
f_{d}\left(x_{0}\right)=0 \quad \text { if } \quad\left|x_{0}\right| \geq d, \\
g(\vec{x})=1 \quad \text { if } \quad|\vec{x}| \leq 1, \\
g(\vec{x})=0 \quad \text { if } \quad|\vec{x}|>1+\delta, \quad 0<\delta<1,
\end{gathered}
$$

with $\vec{J}\left(f \otimes g_{R}\right)$ defined similarly. For other choices, see ref. [35]. The symmetry is characterized by the following property (see [10], p. 111): there exists a one-parameter group of automorphisms $A \mapsto A^{\lambda}$ of $\mathcal{A}_{S}$, strongly continuous with respect to $\lambda$, such that:

(a) if $\mathcal{O}_{L}=\left\{x \in \mathbb{R}^{s+1}|| \vec{x}|+| x_{0} \mid<L\right\}, L>0$, then

$$
A_{L} \in \mathcal{A}_{S}\left(\mathcal{O}_{L}\right) \text { implies } A_{L}^{\lambda} \in \mathcal{A}_{S}\left(\mathcal{O}_{L}\right) ;
$$

(b) if $f_{d}, g$ satisfy (13)-(18) and $J_{0}\left(f_{d} \otimes g_{R}\right)$ is defined by (12), then

$$
\begin{aligned}
& \left.\frac{\mathrm{d}}{\mathrm{d} \lambda}\left(\Omega_{\omega}, A^{\lambda} \Omega_{\omega}\right)\right|_{\lambda=0} \\
& \quad=i \lim _{R \rightarrow \infty}\left(\Omega_{\omega},\left[J_{0}\left(f_{d} \otimes g_{R}\right), A\right] \Omega_{\omega}\right) .
\end{aligned}
$$

Lemma II.2 About the limit on the r.h.s. of (20) we have

$$
\begin{aligned}
\lim _{R \rightarrow \infty} & \left(\Omega_{\omega},\left[J_{0}\left(f_{d} \otimes g_{R}\right), A_{L}\right] \Omega_{\omega}\right) \\
& =\left(\Omega_{\omega},\left[J_{0}\left(f_{d} \otimes g_{R_{1}}\right), A_{L}\right] \Omega_{\omega}\right)
\end{aligned}
$$

if

$$
R_{1} \geq L+d+1 .
$$

The r.h.s. in (21) is independent of the functions $f_{d}$ and $g$, as long as they satisfy (13)-(18).

Proof This follows from local commutativity A7(a), see Lemma 1, pg. 112, of [10].

CRITERION. Given (20), we adopt as in [10] as our criterion for spontaneous breakdown of the symmetry $(s s b)$ associated to the one-parameter group of automorphisms of $\mathcal{A}_{S}$ :

$$
\lim _{R \rightarrow \infty}\left(\Omega_{\omega},\left[J_{0}\left(f_{d} \otimes g_{R}\right), A_{0}\right] \Omega_{\omega}\right)=c \neq 0
$$

for some $A_{0} \in \mathcal{A}_{L}$.

In this paper we shall assume that (23) holds, and derive some constraints from it.

There are two preliminary steps, which we shall prove in this section: firstly, using time translation invariance, which follows from $\mathbf{A 2}$, we show that we may replace $J_{0}\left(f_{d} \otimes g_{R}\right)$ in (23) by a smoothened version, essential to the application of Bogoliubov's inequality in Section III. Secondly, using $\mathbf{A} \mathbf{3}$ we show that $A_{0}$ may be replaced by an average over space-translations, which is essential to relate (23) to the rate of space-like decay of correlations.

Lemma II.3 Assume ssb takes place, in the sense that (23) holds. Then there exists $h \in \mathcal{D}(\mathbb{R})$ such that (23) also holds (with a different $c \neq 0$ ) for the observable

$$
\widetilde{A}_{0}:=\int \mathrm{d} t h(t) \tau_{t}\left(A_{0}\right) .
$$

Proof By Lemma $\amalg .2$

$$
\begin{array}{r}
\lim _{R \rightarrow \infty}\left(\Omega_{\omega},\left[J_{0}\left(f_{d} \otimes g_{R}\right), A_{0}\right] \Omega_{\omega}\right) \\
=\left(\Omega_{\omega},\left[J_{0}\left(f_{d} \otimes g_{R_{1}}\right), A_{0}\right] \Omega_{\omega}\right)
\end{array}
$$

if

$$
R_{1}=L+d+1 .
$$

Now write (25) as

$$
\left(J_{0}\left(f_{d} \otimes g_{R_{1}}\right) \Omega_{\omega}, A_{0} \Omega_{\omega}\right)-\left(A_{0}^{*} \Omega_{\omega}, J_{0}\left(f_{d} \otimes g_{R_{1}}\right) \Omega_{\omega}\right) .
$$

By (26), (27) and A4, and the norm-continuity of the time evolution $\tau_{t}$ as assumed in (i), given $\epsilon>0$, we may choose $h_{\epsilon} \in \mathcal{D}$ such that 


$$
\begin{aligned}
\mid\left(\Omega_{\omega},[\right. & \left.\left.J_{0}\left(f_{d} \otimes g_{R}\right), A_{0}\right] \Omega_{\omega}\right) \\
& -\left(\Omega_{\omega},\left[J_{0}\left(f_{d} \otimes g_{R_{1}}\right), \widetilde{A}_{0}\right] \Omega_{\omega}\right) \mid<\epsilon .
\end{aligned}
$$

See [20], Theorem 4.8, for the proof of (28). The identities (25) and (28) imply

$$
\begin{aligned}
& \lim _{R \rightarrow \infty}\left(\Omega_{\omega},\left[J_{0}\left(f_{d} \otimes g_{R}\right), \widetilde{A}_{0}\right] \Omega_{\omega}\right) \\
& =\left(\Omega_{\omega},\left[J_{0}\left(f_{d} \otimes g_{R_{1}}\right), \widetilde{A}_{0}\right] \Omega_{\omega}\right)=c_{\epsilon} \neq 0 .
\end{aligned}
$$

In the following we ommit the suffixes $\epsilon$ in $h_{\epsilon}$ and $c_{\epsilon}$.

Lemma II.4 Assume ssb takes place, in the sense that (23) holds. Then

$$
\begin{aligned}
\lim _{R \rightarrow \infty} & \left(\Omega_{\omega},\left[J_{0}\left(f_{d} \otimes g_{R}\right), \widetilde{A}_{0}\right] \Omega_{\omega}\right) \\
\quad= & \left(\Omega_{\omega},\left[I_{h}\left(f_{d} \otimes g_{R_{1}}\right), A_{0}\right] \Omega_{\omega}\right) \\
\quad= & c \neq 0
\end{aligned}
$$

where

$$
I_{h}\left(f_{d} \otimes g_{R}\right):=\int \mathrm{d} t h(t) \tau_{-t}\left(J_{0}\left(f_{d} \otimes g_{R}\right)\right) .
$$

$h$ is the function in Lemma II.3 and $R_{1}$ is given by (26).

Proof By A4 and A5,

$$
I_{h}\left(f_{d} \otimes g_{R}\right) \Omega_{\omega}=\int \mathrm{d} t h(t) \mathrm{e}^{-i t L_{\omega}} J_{0}\left(f_{d} \otimes g_{R_{1}}\right) \Omega_{\omega},
$$

since $t \mapsto \mathrm{e}^{-i t L_{\omega}} J_{0}\left(f_{d} \otimes g_{R_{1}}\right) \Omega_{\omega} \in \mathcal{H}_{\omega}$ is continuous, (30) is meaningful and follows from (29) by time translation invariance of $\omega():.=\left(\Omega_{\omega}, . \Omega_{\omega}\right)$.

Our last preliminary lemma makes use of A2:

Lemma II.5 Assume ssb takes place, in the sense that (23) holds. Then, for any $R_{0} \in \mathbb{R}$,

$$
\begin{aligned}
& \lim _{R \rightarrow \infty}\left(\Omega_{\omega},\left[J_{0}\left(f_{d} \otimes g_{R}\right), \widetilde{A}_{0}\right] \Omega_{\omega}\right) \\
& =\left(\Omega_{\omega},\left[I_{h}\left(f_{d} \otimes g_{\widetilde{R}_{0}}\right), A_{R_{0}}\right] \Omega_{\omega}\right)=c \neq 0,
\end{aligned}
$$

where

$$
\widetilde{R}_{0}:=2 R_{0}+L+d+1
$$

and

$$
A_{R_{0}}=\frac{1}{\left|L_{R_{0}}\right|} \int_{L_{R_{0}}} \mathrm{~d}^{s} \vec{x}\left(\sigma_{\vec{x}}\left(A_{0}\right)-\omega\left(A_{0}\right)\right) .
$$

Above, $L_{R_{0}}$ is a s-dimensional region of volume

$$
\left|L_{R_{0}}\right|=O\left(R_{0}^{s}\right)
$$

and $\sigma_{\vec{x}}\left(A_{0}\right) \equiv \pi_{\omega}\left(\sigma_{\vec{x}}\left(A_{0}\right)\right)=\mathrm{e}^{i \vec{P} \vec{x}} \pi_{\omega}\left(A_{0}\right) \mathrm{e}^{-i \vec{P} \vec{x}}$.
Proof Applying Lemma II.4 (30), and space-translation invariance A2,

$$
\begin{aligned}
\lim _{R} \rightarrow \infty & \left(\Omega_{\omega},\left[J_{0}\left(f_{d} \otimes g_{R}\right), \widetilde{A}_{0}\right] \Omega_{\omega}\right) \\
= & \left(\Omega_{\omega},\left[I_{h}\left(f_{d} \otimes g_{R}^{\vec{x}}\right), \sigma_{\vec{x}}\left(A_{0}\right)\right] \Omega_{\omega}\right)
\end{aligned}
$$

for any $\vec{x} \in \mathbb{R}^{s}$, where, by $\mathbf{A} \mathbf{5}$,

$$
g_{R}^{\vec{x}}(\vec{y})=g_{R}(\vec{y}-\vec{x}) .
$$

We assume that as a consequence of finite speed of light, the tiny support of $h$ can be taken into account by slightly increasing $d$. By (37), (38) and Lemma \.2,

$$
\begin{aligned}
\lim _{R \rightarrow \infty}\left(\Omega_{\omega},\left[J_{0}\left(f_{d} \otimes g_{R}\right), \widetilde{A}_{0}\right] \Omega_{\omega}\right) \\
=\left(\Omega_{\omega},\left[I_{h}\left(f_{d} \otimes g_{R_{1}}\right), \sigma_{\vec{x}}\left(A_{0}\right)\right] \Omega_{\omega}\right)
\end{aligned}
$$

as long as

$$
R_{1} \geq 2|\vec{x}|+L+d+1
$$

(33) and (34) follow from (39), (40) and (35).

(33) is the starting point for proving our main results in the next section.

\section{A GOLDSTONE THEOREM IN THERMAL FIELD THEORY}

As a preliminary to our proof of the Goldstone theorem, we write the self-adjoint operator in (33):

$$
J_{0}\left(f_{d} \otimes g_{\widetilde{R}_{0}}\right)=\lim _{n \rightarrow \infty} \lim _{m} \sum_{j=1}^{m} \lambda_{j}^{\prime} E\left(\lambda_{j-1}, \lambda_{j}\right]
$$

where $\lambda_{j}^{\prime} \in\left(\lambda_{j-1}, \lambda_{j}\right]$ is arbitrary,

$$
-n=\lambda_{0}<\lambda_{1}<\ldots<\lambda_{m}=n
$$

and $\lim _{m}$ is the limit when $\max \left\{\left|\lambda_{j}-\lambda_{j-1}\right| \mid j=\right.$ $1, \ldots, m\}$ tends to zero. This is the spectral theorem (see, e.g., 21], p. 342). We shall abbreviate the double limit in (41) by $n, m \rightarrow \infty$, and denote the finite sum $\sum_{j=1}^{m} \lambda_{j}^{\prime} \cdot E\left(\lambda_{j-1}-\lambda_{j}\right]$ by $J_{0}^{n, m}\left(f_{d} \otimes g_{R_{1}}\right)$. Above,

$$
E\left(\lambda_{j-1}, \lambda_{j}\right]=E\left(-\infty, \lambda_{j}\right]-E\left(-\infty, \lambda_{j-1}\right]
$$

are the (right-continuous) spectral projections associated to $J_{0}$. By $\mathbf{A} \mathbf{8}$ et seq., they belong to $\mathcal{R}(\mathcal{O})$, and thus $J_{0}^{n, m}\left(f_{d} \otimes g_{\widetilde{R}_{0}}\right) \in \mathcal{R}(\mathcal{O})$ for any finite $n, m$. The limit (41) is understood to be acting on any vector in the domain of $J_{0}\left(f_{d} \otimes g_{\widetilde{R}_{1}}\right)$.

In correspondence to (33) and (41), we define

$$
I_{h}^{n, m}:=\int \mathrm{d} t h(t) \tau_{-t}\left(J_{0}^{n, m}\left(f_{d} \otimes g_{\widetilde{R}_{0}}\right)\right) .
$$

By definition (44), $I_{h}^{n, m}$ is a smooth element of $\mathcal{R}(\mathcal{O})$ (ignoring once again the fact that one has to increases the 
region $\mathcal{O}$ by a small amount in order to accommodate for the spreading due to the convolution with $h$ ), and thus, by (iii), belongs to $\mathcal{A}_{S}(\mathcal{O})$. We now apply Corrollary V.2 of Appendix A, with $C=I_{h}^{n, m}, A=A_{R_{0}}=A_{R_{0}}^{*}$ (this may be assumed without loss of generality, otherwise we may decompose $A=S+i T$ with $S=S^{*}, T=T^{*}$. This leads to imaginary and real parts of $c$ in (33).), and obtain from the Bogoliubov's inequality (86):

$$
\begin{aligned}
& \frac{1}{\beta}\left|\omega\left(\left[I_{h}^{n, m}, A_{R_{0}}\right]\right)\right|^{2} \\
& \quad \leq \omega\left(\left[I_{h}^{n, m},\left.i\left(\frac{\mathrm{d}}{\mathrm{d} t} \tau_{t}\left(I_{h}^{n, m}\right)\right)\right|_{t=0}\right]\right) \omega\left(A_{R_{0}}^{2}\right) .
\end{aligned}
$$

Lemma III.1 Under the assumptions made,

$$
\begin{aligned}
\lim _{n, m} \rightarrow \infty & \omega\left(\left[I_{h}^{n, m}, A_{R_{0}}\right]\right) \\
& =\omega\left(\left[I_{h}\left(f_{d} \otimes g_{\widetilde{R}_{0}}\right), A_{R_{0}}\right]\right),
\end{aligned}
$$

and

$$
\begin{aligned}
& \lim _{n, m \rightarrow \infty} \omega\left(\left[I_{h}^{n, m},\left.i\left(\frac{\mathrm{d}}{\mathrm{d} t} \tau_{t}\left(I_{h}^{n, m}\right)\right)^{*}\right|_{t=0}\right]\right) \\
& \left.=i \omega\left(\left[I_{h}\left(f_{d} \otimes g_{\widetilde{R}_{0}}\right), \int \mathrm{d} t h^{\prime}(t) J_{0}\left(f_{d}(\cdot-t)\right) \otimes g_{\widetilde{R}_{0}}\right)\right]\right) .
\end{aligned}
$$

Proof By A4, A5 and the spectral theorem,

$$
\begin{aligned}
& \mathrm{e}^{i t L_{\omega}} J_{0}^{n, m}\left(f_{d} \otimes g_{\widetilde{R}_{0}}\right) \Omega_{\beta} \\
& \left.\underset{n, m \rightarrow \infty}{\longrightarrow} J_{0}\left(f_{d}(\cdot-t)\right) \otimes g_{\widetilde{R}_{0}}\right) \Omega_{\beta}
\end{aligned}
$$

uniformly in $t \in \mathbb{R}$. Hence, by (44),

$$
\begin{aligned}
& I_{h}^{n, m} \Omega_{\omega}=\int \mathrm{d} t h(t) \mathrm{e}^{i t L_{\omega}} J_{0}^{n, m}\left(f_{d} \otimes g_{\widetilde{R}_{0}}\right) \Omega_{\omega} \\
& \left.\underset{n, m \rightarrow \infty}{\longrightarrow} \int \mathrm{d} t h(t) J_{0}\left(f_{d}(\cdot-t)\right) \otimes g_{\widetilde{R}_{0}}\right) \Omega_{\omega}
\end{aligned}
$$

from which (46) follows. Again by (44),

$$
\left.\left(\frac{\mathrm{d}}{\mathrm{d} t} \tau_{t}\left(I_{h}^{n, m}\right)\right)\right|_{t=0}=\int \mathrm{d} t h^{\prime}(t) \tau_{t}\left(J_{0}^{n, m}\left(f_{d} \otimes g_{\widetilde{R}_{0}}\right)\right) .
$$

We obtain (47) from (37) by the same argument leading from (48) to (49).

We now use assumption $\mathbf{A} \mathbf{1}$, that $\omega$ is a factor state.

Lemma III.2 Let $A, B \in \mathcal{A}_{S}$. Then

$$
F_{A, B}(\vec{x}):=\left(\omega\left(A \sigma_{\vec{x}}(B)\right)-\omega(A) \omega(B)\right) \underset{|\vec{x}| \rightarrow \infty}{\longrightarrow} 0 .
$$

Proof This follows from A1, A2 and (4) of (iv), see [18], Theorem 3.2.2.

We are now able to state and prove our main result. Assume, with (51), that

$$
F_{A, B}(\vec{x})=O\left(|\vec{x}|^{-\delta}\right)
$$

as $|\vec{x}| \rightarrow \infty$. Our thermal Goldstone theorem relates the rate of clustering $\delta$ in (52) to $s s b$ :
Theorem III.3 (Thermal Goldstone theorem). Let a relativistic quantum field theory be defined as a $C^{*}$ algebraic dynamical system $\left(\mathcal{A}_{S}, \omega, \tau\right)$, satisfying $(i)-(i v)$ as well as $\mathbf{A 1 - A 8}$. Then, if $s \geq 3$ and if there is ssb as defined by (23), the rate of clustering $\delta$ in (52) must satisfy

$$
\delta \leq s-2
$$

Proof By Lemma II.5, (23) implies (33), (34), which, by (45) and Lemma III.1 leads to the inequality, for any $R_{0} \in \mathbb{R}$ :

$$
\begin{aligned}
\frac{1}{\beta}\left|\omega\left(\left[I_{h}\left(f_{d} \otimes g_{\widetilde{R}_{0}}\right), A_{R_{0}}\right]\right)\right|^{2} & \\
\leq i \omega\left(A_{R_{0}}^{2}\right) \omega\left(\left[I_{h}\left(f_{d} \otimes g_{\widetilde{R}_{0}}\right),\right.\right. & \left.\left.\quad \int \mathrm{d} t h^{\prime}(t) J_{0}\left(f_{d}(\cdot-t) \otimes g_{\widetilde{R}_{0}}\right)\right]\right) .
\end{aligned}
$$

By A6 (local current conservation)

$$
\begin{aligned}
& \left(\int \mathrm{d} t h^{\prime}(t) J_{0}\left(f_{d}(\cdot-t)\right) \otimes g_{\widetilde{R}_{0}}\right) \\
& \left.\left.\quad+\int \mathrm{d} t h(t) \vec{J}\left(f_{d}(\cdot-t)\right) \otimes \nabla g_{\widetilde{R}_{0}}\right)\right) \Omega_{\omega}=0 .
\end{aligned}
$$

In (55) we applied $\mathbf{A 6}$ to the function $f=\left(h \star f_{d}\right) \otimes g_{R_{0}}$, where the asterisk denotes convolution.

Inserting (55) into (54) we are led to find a bound to the quantity

$$
\begin{array}{r}
M:=i \omega\left(\left[\int \mathrm{d} t_{1} h\left(t_{1}\right) \vec{J}\left(f_{d}\left(\cdot-t_{1}\right)\right) \otimes g_{\widetilde{R}_{0}}\right),\right. \\
\left.\left.\left.\int \mathrm{d} t_{2} h\left(t_{2}\right) \vec{J}\left(f_{d}\left(\cdot-t_{2}\right)\right) \otimes \nabla g_{\widetilde{R}_{0}}\right)\right]\right) .
\end{array}
$$

By (12) and (17), (18),

$$
\frac{\partial g_{\widetilde{R}_{0}}}{\partial x_{i}}=\frac{1}{\widetilde{R}_{0}} \frac{\partial g}{\partial x_{i}}\left(\frac{\vec{x}}{\widetilde{R}_{0}}\right) \quad i=1,2, \ldots, s,
$$

where

$$
\left(\frac{\partial g}{\partial x_{i}}\right)\left(\frac{\vec{x}}{\widetilde{R}_{0}}\right)=0 \text { if }|\vec{x}| \leq \widetilde{R}_{0} \text { and }|\vec{x}|>\widetilde{R}_{0}+\delta
$$

and thus

$$
\operatorname{supp}\left(\frac{\partial g}{\partial x_{i}}\right)\left(\frac{\vec{x}}{\widetilde{R}_{0}}\right) \subseteq \Gamma_{\widetilde{R}_{0}}
$$

where, by (58),

$$
\Gamma_{\widetilde{R}_{0}}=\left\{\vec{x} \in \mathbb{R}^{s}\left|\widetilde{R}_{0} \leq\right| \vec{x} \mid \leq \widetilde{R}_{0}+\delta\right\}
$$

is a region of volume

$$
\left|\Gamma_{\widetilde{R}_{0}}\right|=\left|S_{\mathcal{S}}\right|\left[\left(R_{0}+\delta\right)^{s}-R_{0}^{s}\right]=O\left(\widetilde{R}_{0}^{s-1}\right)
$$

with $\left|S_{\mathcal{S}}\right|$ the volume of a $s$-dimensional sphere of unit radius. Let $\Gamma_{\widetilde{R}_{0}}^{i n t}$ denote the interior of $\Gamma_{\widetilde{R}_{0}}$. We consider the cover (see Theorem VI.1 of Appendix B):

$$
\Gamma_{\widetilde{R}_{0}}^{i n t}=\bigcup_{i \in I} G_{i}
$$


where

$$
|I|=O\left(\widetilde{R}_{0}^{s-1}\right)
$$

and $G_{i}$ are open hypercubes of side $(1+\epsilon), 0<\epsilon<1$, in $\mathbb{R}^{s}$, there being only $O(1)$ such hypercubes along a radius, in accordance to (59): in (63), $|I|$ is the cardinality of the set $I$. This is of course, only one possible choice for the cover (62). In correspondence to the latter, we write now the second term in (56) following the theorem on the partition of unity in Appendix B: let

$$
\beta_{i}=\sum_{j \in J} \alpha_{j}, \quad \operatorname{supp} \alpha_{j} \in G_{i},
$$

corresponding to $\mathbf{B 1}$; by $\mathbf{B 2}$ and $\mathbf{B 3}, 0 \leq \beta_{i} \leq 1$ for all $i$.

We define

$$
i \in I \mapsto \gamma_{i}^{r}:=\beta_{i} \frac{\partial g\left(\vec{x} / \widetilde{R}_{0}\right)}{\partial x_{r}} .
$$

Then, by (57)- (65):

$$
\begin{aligned}
& \int \mathrm{d} t_{2} h\left(t_{2}\right) \vec{J}\left(f_{d}\left(\cdot-t_{2}\right) \otimes \nabla g_{\widetilde{R}_{0}}\right) \\
& =\frac{1}{\widetilde{R}_{0}} \sum_{r=1}^{s} \sum_{i \in I} \int \mathrm{d} t_{2} h\left(t_{2}\right) J_{r}\left(f_{d}\left(\cdot-t_{2}\right) \otimes \gamma_{i}^{r}\right) .
\end{aligned}
$$

By locality A7b together with (66), we have, for $M$ defined by (56):

$$
\begin{array}{r}
M=\frac{i}{\widetilde{R}_{0}} \sum_{r=1}^{s} \sum_{i \in I} \omega\left(\left[\int \mathrm{d} t_{1} h\left(t_{1}\right) J_{0}\left(f_{d}\left(\cdot-t_{1}\right) \otimes g_{R_{i}}\right),\right.\right. \\
\left.\left.\int \mathrm{d} t_{2} h\left(t_{2}\right) J_{r}\left(f_{d}\left(\cdot-t_{2}\right) \otimes \gamma_{i}^{r}\right)\right]\right),
\end{array}
$$

where

$$
R_{i}=O(1) \quad \forall i \in I,
$$

is the minimal length such that $\operatorname{supp} f_{d}\left(\cdot-t_{1}\right) \otimes g_{R_{i}}$ is time-like to $\operatorname{supp} f_{d}\left(\cdot-t_{2}\right) \otimes \gamma_{i}^{r}$ : it depends only on $d$, the support of $h$ and the diameter of the support of $\gamma_{i}^{r}$, which is of order one by our choice of $G_{i}$ in (62). By (63), (67), 68) and Assumption A4

$$
0 \leq M \leq \text { const. } \widetilde{R}_{0}^{s-2},
$$

where the constant is independent of $\widetilde{R}_{0}$. By (24), (35), (36) and the KMS condition (Assumption A2)

$$
\begin{aligned}
\omega\left(A_{R_{0}}^{2}\right)= & \frac{1}{\left.L_{R_{0}}\right|^{2}} \int_{L_{R_{0}}} \mathrm{~d}^{3} \vec{x} \int_{L_{R_{0}}} \mathrm{~d}^{3} \vec{y} \\
& \times\left(\omega\left(A_{0} \sigma_{\vec{x}-\vec{y}}\left(A_{0}\right)\right)-\omega\left(A_{0}\right)^{2}\right) \\
\leq & \frac{c}{R_{0}^{s}}\left(2 R_{0}\right)^{s-\delta}=c R_{0}^{-\delta} .
\end{aligned}
$$

Inserting (54), (56), (69) and (70) into (33) of Lemma II.5) we obtain, with (34):

$$
0 \neq c \leq d \cdot R_{0}^{s-2-\delta},
$$

where $d$ is a positive constant independent of $R_{0}$. (71) is true for any $R_{0} \in \mathbb{R}$; taking $R_{0} \rightarrow \infty$, we obtain a contradiction unless (53) holds.
We now remark on the restriction to $s \geq 3$ in Theorem III.3. There are no finite-temperature equilibrium twopoint functions (with vanishing chemical potential) for the massless free field for $s=1$ and $s=2$ and nothing is known for interacting theories (see, e.g., [34], pp. 144 and 151 for a pedagogic discussion). The proof of Theorem II.3 does not work for $s=1$ (the surface degenerates to a point) and does work for $s=2$ but the result is inconclusive, although (53) correctly predicts a borderline behaviour of the case $s=2$.

For the scalar free field of mass $m$, the two point function corresponding to $F_{A, B}$ in (51) is, for $s=3$ :

$$
\begin{aligned}
W_{\beta}(x, m)=(2 \pi)^{-3} \int \mathrm{d}^{4} p & \epsilon\left(p_{0}\right) \delta\left(p^{2}-m^{2}\right) \\
& \times\left(1-\mathrm{e}^{-\beta p_{0}}\right)^{-1} \mathrm{e}^{-i p x} \\
= & (2 \pi)^{-3} \int \frac{\mathrm{d}^{3} \vec{p}}{2 \omega_{\vec{p}}} \mathrm{e}^{i \vec{p} \vec{x}}\left(\frac{\mathrm{e}^{-i \omega_{\vec{p}} x_{0}}}{1-\mathrm{e}^{-\beta \omega_{\vec{p}}}}+\frac{\mathrm{e}^{i \omega_{\vec{p}} x_{0}}}{\mathrm{e}^{\beta \omega_{\vec{p}}}-1}\right)
\end{aligned}
$$

where

$$
\omega_{\vec{p}}:=\left(\vec{p}^{2}+m^{2}\right)^{1 / 2} .
$$

For $m=0$ the asymptotic behaviour of $W_{\beta}(x, m)$ for $\left|x_{0}\right| \ll|\vec{x}|$ is seen from (72) to be the same as that of

$$
\int \frac{\mathrm{d}^{3} \vec{p}}{|\vec{p}|^{2}} \mathrm{e}^{i \vec{p} \vec{x}} \cong \frac{1}{|\vec{x}|},
$$

which contrasts with the $\frac{1}{|\vec{x}|^{2}}$ fall-off in the massless $T=0$ case. (74) is also the asymptotic rate of fall-off of $F_{A, B}(\vec{x})$ in (51) in the free massless case, and thus the result of Theorem III.3 may also be expected to be optimal in thermal (relativistic) quantum field theory, as it is in nonrelativistic quantum statistical mechanics (see [5, 7]).

We conclude this section with some results and conjectures related to Theorem III.3, which help to clarify its significance. The conjectured optimality of Theorem III.3 suggests the more precise:

\section{Conjecture III.4}

(i) in the massless case $(m=0)$

$$
O\left(|\vec{x}|^{-\delta}\right) \quad \text { with } \quad \delta \leq 1
$$

(ii) in the massive case $(m>0)$

$$
O\left(|\vec{x}|^{-\delta}\right) \quad \text { with } \quad \delta>1 .
$$

Corollary III.5 Under Conjecture III.4 ssb of a continuous internal symmetry in thermal relativistic quantum field theory with a conserved local current implies the existence of zero mass particles in the theory.

Thus, under Conjecture III.4 the statement of Goldstone's theorem for $T>0$ is the same as the corresponding one for $T=0$ (see [10] 11]). 
Remark III.1 A certain form of slow decay in spacelike directions has also been proved in [G] to be necessary for the existence of $\mathrm{ssb}$ at $T>0$ (see (18) of [G]).

Remark III.2 Note that (76) does not assume exponential decay in the $m>0$ case - as happens in the free field case (72). This is in agreement with the conjectured behaviour of the damping form factors in [24] (see also the discussion in [1]]).

Is there a spectral theoretic statement related to (75) and (76)? Since the spectrum $\sigma\left(L_{\omega}\right)$ of $L_{\omega}$ is the whole real line,

$$
\sigma\left(L_{\omega}\right)=\mathbb{R}
$$

this question has no obvious answer. However, if $\Omega_{\omega}$ is the unique (up to a phase) normalised eigenvector of $L_{\omega}$ with eigenvalue 0 , then $\omega$ is a factor state 25] and one has the following result (see [1, 30]):

Theorem III.6 Let $\Omega_{\omega}$ be as above, and $P^{+}$denote the projection onto the strictly positive part of $\sigma\left(L_{\omega}\right)$. Assume there exist positive constants $\delta>0$ and $C_{1}(\mathcal{O})$ such that

$$
\left\|\mathrm{e}^{-\lambda L_{\omega}} P^{+} \pi_{\omega}(A) \Omega_{\omega}\right\| \leq C_{1}(\mathcal{O}) \lambda^{-\delta}\|A\|
$$

for all $A \in \mathcal{A}(\mathcal{O})$. Consider now two space-like separated space-time regions $\mathcal{O}_{1}, \mathcal{O}_{2}$, which can be embedded into $\mathcal{O}$ by translation and such that $\mathcal{O}_{1}+r e \subset \mathcal{O}_{2}^{\prime}, r \gg \beta$; then, for all $A \in \mathcal{A}\left(\mathcal{O}_{1}\right)$, and all $B \in \mathcal{A}\left(\mathcal{O}_{2}\right)$

$$
|\omega(B A)-\omega(B) \omega(A)| \leq C_{2} r^{-2 \delta}\|A\|\|B\| .
$$

The constant $C_{2}(\beta, \mathcal{O}) \in \mathbb{R}^{+}$may depend on the temperature $T=\beta^{-1}$ and the size of the region $\mathcal{O}$, but is independent of $r, A$, and $B$.

As remarked in [1], from explicit calculations one expects that $\delta=1 / 2$ for free massless bosons in $3+1$ spacetime dimensions, and thus the exponent on the r.h.s. of (79) is optimal due to (74).

It is interesting that, in the massive case, for $T=0$, exponential decay on the r.h.s. of (79) follows from the spectral gap in $H_{\omega}>0$, i.e., exponential decay in $\lambda$ of

$$
\left\|\mathrm{e}^{-\lambda H_{\omega}} \pi_{\omega}(A) \Omega_{\omega}\right\|,
$$

by the cluster theorem [26], while, for $T>0$, sufficiently fast polynomial decay of correlations - (79), with $\delta>$ $1 / 2$ - equally follows from sufficiently fast decay of

$$
\left\|\mathrm{e}^{-\lambda L_{\omega}} P^{+} \pi_{\omega}(A) \Omega_{\omega}\right\|,
$$

- (78) with $\delta>1 / 2-$ if (76) is correct. It is to be remarked that (79) is related (see 29]) to the BuchholzWichmann nuclearity property [28].

\section{DISCUSSION AND OUTLOOK}

In this paper we have shown that a Goldstone theorem may be proved in thermal quantum field theory, relating $s s b$ to the space-like decay of the two-point function (Theorem III.3 of Section (III). Since the limiting behaviour (53) of Theorem III.1 agrees with that of the massless free field theory (74), we were led to the conjecture that the theorem may be optimal, as occurs in non-relativistic quantum statistical mechanics, leading to a sharp distinction (75), (76) between massive and massless thermal rqft. The latter is found by examining the rate of fall-off of the two-point function only in space-like directions. If this conjecture is correct, Corollary III.5 provides a statement of Goldstone's theorem for $T>0$, which is quite analogous to the one for $T=0$ (see [10, 11]).

We have chosen to set our scale large only as far as space-like distances are concerned. As remarked in [27], this may be appropriate for discussing global issues like superselection sectors, statistics and symmetries. But there remains scattering theory with the associated notions of particles and infraparticles, and there large timelike distances are crucial. Thus, if one is really concerned with unravelling the concept of particle in thermal rqft, the approach of Bros and Buchholz $([9,23])$ is the most natural one. However, time-like decay as $|t|^{-3 / 2}$ for $\vec{x}=\vec{v} t$ (which follows from (72)) leads, together with the assumption of a sharp dispersion law, to the famous Narnhofer-Requardt-Thirring theorem [30], according to which there is no interaction. We refer to 24] (see Sect. d., p. 518) for a lucid discussion of possible ways out of this dilemma, but the matter still remains under discussion.

A relevant open problem is a purely algebraic version of the Goldstone theorem in the case of positive temperature, in analogy to what was accomplished in ref. [36] for the ground state. It should also be remarked that domain problems such as the one pertaining to assumption A4 have been solved in [36], without the need of any assumption, in a very ingenious way (see (3.6) et seq), but we were unable to do the same here. In addition, nonconserved currents, successfully dealt with in [36], remains an open problem for $T>0$. Finally, ssb of Lorentz and Galilei symmetries has been studied by a different method in ref. [37], where references to related work by Requardt are to be found.

A different but fundamental set of issues related to time-like clustering, not mentioned in [27], concerns stability. The time-like cluster property (also called mixing property [25]

$$
\lim _{t \rightarrow \infty}\left(\omega\left(A \tau_{t}(B)\right)-\omega(A) \omega(B)\right)=0
$$

implies, for $T>0$, the dynamic stability condition of Haag, Kastler and Trych-Pohlmeyer 31]

$$
\lim _{T \rightarrow \infty} \int_{-T}^{T} \mathrm{~d} t \omega\left(\left[A, \tau_{t}(B)\right]\right)=0
$$


(see [17], Vol. 2, Theorem 5.4.12, pg. 165). Although (82) has been proved for the ground state of relativistic quantum field theories [32], it is still open for thermal KMS states, although a similar property has been proved for a weakly dense set of (in general non-KMS) states [33]. Proof of (82) for KMS states would imply the property of return to equilibrium 25], as well as the dynamic stability condition (83), both quite deep, and in general, hard to prove (see [25] for references).

Acknowledgements. This paper originates from discussion during our stay at the Erwin Schrödinger Institute (ESI) Vienna, from 1-14 June 2009. We are grateful to Prof. J. Yngvason for making our visit possible, as well as to Heide Narnhofer and Geoffrey Sewell for several enjoyable discussions on related matters. We also thank Heide Narnhofer for remarks on the manuscript, as well as Manfred Requardt for letting us know of ref [37].

\section{APPENDIX A}

Theorem V.1 Let $\mathcal{A}$ be a $C^{*}$-algebra and $\omega$ a state on $\mathcal{A}$ satisfying the KMS condition (5) w.r.t. a group of normcontinuous automorphisms $\left\{\tau_{t}\right\}_{t \in \mathbb{R}}$. Let $A \in \mathcal{A}$ and $C \in$ $\mathcal{A}$ be both of the form

$$
C=\int \mathrm{d} t h(t) \tau_{t}(B)
$$

with some $B \in \mathcal{A}$ and

$$
\hat{h} \in \mathcal{D}=C_{0}^{\infty}(\mathbb{R}),
$$

where $\hat{h}$ denotes Fourier transform of $h$. Then

$\frac{2}{\beta}\left|\omega\left(\left[C, A^{*}\right]\right)\right|^{2} \leq \omega\left(\left[C,\left.i\left(\frac{\mathrm{d}}{\mathrm{d} t} \tau_{t}(C)\right)\right|_{t=0}\right]\right) \cdot \omega\left(\left\{A, A^{*}\right\}\right)$,

where $\{A, B\}:=A B+B A$.

Inequality (86) (Bogoliubov's inequality) may be extended to all $A \in \mathcal{A}$ and to those $C$ of the form

$$
C=\int \mathrm{d} t g(t) \tau_{t}(B)
$$

where $g \in C^{\infty}(\mathbb{R})$ is such that, given any $\epsilon>0$, there exists $h$ satisfying (85) such that

$$
\int \mathrm{d} t\left|h^{\prime}(t)-g^{\prime}(t)\right|<\epsilon
$$

and

$$
\int \mathrm{d} t|h(t)-g(t)|<\epsilon
$$

Proof See [14] and [17], Vol. II, pg. 333. Normcontinuity of the time-translation automorphisms was not explicitly stated in [14], but is used to extend the result from $A$ in the class $(84)$, (85) to the whole of $\mathcal{A}$ by density (see [20], Theorem 4.8). The extension to (87) was not mentioned in [14], but follows from (84), (85), (86), (87) and (88), together with

$$
\left.\frac{\mathrm{d}}{\mathrm{d} t} \tau_{t}(C)\right|_{t=0}=\int \mathrm{d} t g^{\prime}(t) \tau_{t}(B)
$$

for $C$ of the form (87), and

$$
\left.\frac{\mathrm{d}}{\mathrm{d} t} \tau_{t}(C)\right|_{t=0}=\int \mathrm{d} t h^{\prime}(t) \tau_{t}(B)
$$

for $C$ of the form (84).

Corollary V.2 Let $A \in \mathcal{A}_{S}$ (see Section II) and $C$ be of the form (87), with $B \in \mathcal{A}_{S}$ and

$$
g \in C_{0}^{\infty}(\mathbb{R})
$$

Then, if $\omega$ is a state on $\mathcal{A}_{S}$ satisfying the $K M S$ condition, Condition A3 holds.

Proof Since $h \in \mathcal{S}(\mathbb{R})$ by (85), given $g$ satisfying (92), we may choose the 'tail to infinity' in $h$ appropriately so that (88) and (89) hold.

Remark V.1 We use (86) in the main text under conditions of Corollary V.2. Since conditions (85) and (92) are mutually excludent by the Paley-Wiener theorem (see e.g. [21], Exercise 8 of Chap. 10), the density argument in Theorem V.1 is important for the application we make of (86) in Section III.

Remark V.2 For the proof of positivity of the middle term in (85) and other questions related to the Bogoliubov scalar product, see ([17], Vol. II, pg. 334). For some inequalities in statistical mechanics for $W^{*}$ systems, see [22].

\section{APPENDIX B}

We state here, for the reader's convenience, the theorem (partition of unity) used in Theorem III.3 of Section III.

Theorem VI.1 (see [23], Theorem, p. 61, Chap. I, Sect. 12). Let $G$ be an open set of $\mathbb{R}^{n}$, and let a family of open sets $\left\{G_{i} \mid i \in I\right\}$ cover $G$, i.e., $G=\bigcup_{i \in I} G_{i}$. Then there exists a system of functions $\left\{\alpha_{j}(x) \mid j \in J\right\}$ of $C_{0}^{\infty}\left(\mathbb{R}^{n}\right)$ such that

B1 for every $j \in J, \operatorname{supp}\left(\alpha_{j}\right)$ is contained in some $G_{i}$;

B2 for every $j \in J$, the function $\alpha_{j}$ satisfies $0 \leq$ $\alpha_{j}(x) \leq 1$ for all $x \in \mathbb{R}^{n}$

B3 $\sum_{j \in J} \alpha_{j}=1$ for $x \in G$. 
[1] C. Jäkel, Thermal quantum field theory, published in: Encyclopedia of Mathematical Physics, Elsevier, Editors: Jean-Pierre Francoise, Gregory Naber, Tsou Sheung Tsun, ACADEMIC PRESS (2006) ISBN: 0-12-512660-3.

[2] N.P. Landsman and Ch.G. van Weert, Real- and imaginary-time field theory at finite temperature and density, Phys. Rep. 145, 141-249 (1987).

[3] D. Buchholz, I. Ojima and H. Roos, Thermodynamic properties of non-equilibrium states in quantum field theory, Ann. Phys. 297, 219-242 (2002).

[4] C. Dappiaggi, K. Fredenhagen and N. Pinamonti, Stable cosmological models driven by a free quantum scalar field, Phys. Rev. D 77 (2008) 104015.

[5] W.F. Wreszinski, Charges and symmetries in quantum theories without locality, Fortschritte der Physik 35, 379413 (1987).

[6] L.J. Landau, J. F. Perez and W.F. Wreszinski, Energy gap, clustering and the Goldstone theorem in quantum statistical mechanics, J. Stat. Phys. 26, 755-766 (1981).

[7] P.A. Martin, A remark on the Goldstone theorem in statistical mechanics, Nuovo Cimento 68B, 302-314 (1982)

[8] D. Mermin and H. Wagner, Absence of ferromagnetism or antiferromagnetism in one- or two-dimensional isotropic Heisenberg models, Phys. Rev. Lett. 17, 1133-1136 (1966); see also D. Ruelle, Statistical Mechanics: Rigorous Results, Addison-Wesely Publishing Co., Inc. (1969) and (1989).

[9] J. Bros and D. Buchholz, The unmasking of thermal Goldstone bosons, Phys. Rev. D58, 125012 (1998).

[10] D. Kastler, D.W. Robinson and J.A. Swieca, Conserved currents and associated symmetries; Goldstone's theorem, Commun. Math. Phys. 2, 108-120 (1966).

[11] H. Ezawa and J.A. Swieca, Spontaneous breakdown of symmetries and zero-mass states, Commun. Math. Phys. 5, 330-336 (1967).

[12] F.J. Dyson, E. Lieb, and B. Simon, Phase transitions in quantum spin systems with isotropic and non-isotropic interactions, J. Stat. Phys. 18, 335-383 (1978).

[13] J.A. Swieca, Goldstone's theorem and related topics, Cargése Lectures in Physics, Ed. D. Kastler, Gordon and Breach (1970).

[14] J.C. Garrison and J. Wong, Bogoliubov inequalities for infinite systems, Commun. Math. Phys. 26, 1-5 (1972).

[15] J. Naudts, A. Verbeure and R. Weder, Linear response theory and kms condition, Commun. Math. Phys. 44, 8799 (1975).

[16] G. Roepstorff, Correlation inequalities in quantum statistical mechanics and their application in the Kondo problem, Commun. Math. Phys. 46, 253-262 (1976).

[17] O. Bratteli and D.W. Robinson, Operator Algebras and Quantum Statistical Mechanics Vol. I,II, SpringerVerlag, New York-Heidelberg-Berlin (1981).

[18] R. Haag, Local Quantum Physics: Fields, Particles, Algebras, Springer-Verlag, Berlin-Heidelberg-New York, 2nd revised and enlarged edition (1996).
[19] R.V. Kadison and J.R. Ringrose, Fundamentals of the Theory of Operator Algebras, Vol. 2, Academic Press (1986) ISBN 0123933021.

[20] N.M. Hugenholtz, States and representations in statistical mechanics, published in: Mathematics of contemporary physics, Streater (Ed.), p. 145., Academic Press, London, (1972).

[21] Ph. Blanchard and E. Bruening, Mathematical Methods in Physics, Birkhäuser Boston (2002) ISBN-10: 0817642285, ISBN-13: 978-0817642280.

[22] Ruskai, M.B., Inequalities for traces on von Neumann algebras, Commun. Math. Phys. 26, 280-289 (1972).

[23] K. Yoshida, Functional Analysis and Its Applications, Springer-Verlag, New York (1971).

[24] J. Bros and D. Buchholz, Axiomatic analyticity properties and representations of particles in thermal quantum field theory, Ann. Inst. H. Poincaré 64, 495-521 (1996).

[25] C.A. Pillet, Quantum Dynamical Systems, in S.Attal, A. Joye and C.A. Pillet, eds, Open Quantum Systems I, the Hamiltonian Approach, vol. 1880 of Lecture Notes in Mathematics, Springer-Verlag, New York 2006.

[26] K. Fredenhagen, A remark on the cluster theorem, Commun. Math. Phys. 97, 461-463 (1985).

[27] R. Brunetti and K. Fredenhagen, Algebraic Approach to Quantum Field Theory, published in: Encyclopedia of Mathematical Physics, Elsevier, Editors: Jean-Pierre Francoise, Gregory Naber, Tsou Sheung Tsun, ACADEMIC PRESS (2006) ISBN: 0-12-512660-3.

[28] D. Buchholz and E. Wichmann, Causal independence and the energy level density of states in local quantum field theory, Commun. Math. Phys. 106, 321-344 (1986).

[29] C.D. Jäkel, Decay of spatial correlations in thermal states, Ann. Inst. Henri Poincaré 69, 425 (1998).

[30] H. Narnhofer, M. Requardt and W. Thirring, Quasiparticles at finite temperatures, Commun. Math. Phys. 92, 247-268 (1983).

[31] R. Haag, D. Kastler, and E.B. Trych-Pohlmeyer, Stability and equilibrium states, Commun. Math. Phys. 38, 173193 (1974), see also: R. Haag and E.B. Trych-Pohlmeyer, Stability properties of equilibrium states, Commun. Math. Phys. 56, 213-224 (1977).

[32] D. Maison, Eine Bemerkung zu Clustereigenschaften, Comm. Math. Phys. 10, 48-51 (1968).

[33] C.D., Jäkel, H., Narnhofer, and W.F., Wreszinski, On the mixing property for a class of states of relativistic quantum fields.

[34] S. A. Fulling and S. N. M. Ruijsenaars, Temperature, periodicity and horizons, Phys. Rep. 152, 135-176 (1987).

[35] M. Requardt, Symmetry Conservation and Integrals over Local Charge Densities in Quantum Field Theory, Comm. Math. Phys. 50, 259-263 (1976).

[36] D. Buchholz, S. Doplicher, R. Longo and J.E. Roberts, A New Look at Goldstone's Theorem, Rev. Math. Phys. (Special Issue) 49-83 (1992).

[37] M. Requardt, Spontaneous Symmetry Breaking of Lorentz and (Galilei) Boosts in (Relativistic) Many Body Systems, arXiv 0805.3022 (2008). 